\title{
Detecting and determining self-incompatibility alleles in almond genotypes using molecular method
}

\author{
Professor Dr. Sorush Niknamian \\ Board Member of Weston A Price Foundation, Washington DC, USA \\ Email: so.niknamian@gmail.com
}

\begin{abstract}
One of the problems in almond production is self-incompatibility in this plant, which is considered as an important improvement point for this tree. Self-incompatibility causes non-uniformity and garden management problems. Most cultivars of almonds have gametophytic self-incompatibility that is controlled by a multi-allelic gene site. The inoculation inhibitor factor in this inhibitory system is the stop of pollen tube growth in the style. This study aims to detect and determine the self-compatible genotype from among the studied samples and determine the self-incompatibility alleles in the studied masses. For the experiment, the leaf samples were collected from 100 almond genotypes that had good products in recent years. The DNA of young leaf samples in these genotypes was extracted using Gept and Celeg (1989) method with a few changes. Today, various methods have been invented for detecting the genotypes and self-compatible cultivars from selfincompatible cultivars as well as S alleles in almonds, including the PCR method. Therefore, in order to detect $\mathrm{S}$ alleles in different almond and some hybrid genotypes, the exclusive primer pairs, including AS1II-AmyC5R, ConF-ConR and Cebador2-Cebador8, were used in the polymerase chain reaction. All of the primers have been used by other researchers to detect almond alleles and the effectiveness of these pairs of primers was confirmed in this experiment. Using the AS1IIAmyC5R and Cebador2-Cebador8 primers, the $S_{f}$ allele with the size of 1200 base pairs was detected. Using the ConF-ConR pair of primer, the $S_{1}, S_{2}, S_{3}, S_{10}, S_{11}, S_{23}$, and $S_{31}$ alleles were detected in the self-incompatible samples. Using AS1II-AmyC5R pair of primer, the known alleles of $S_{3}, S_{f}, S_{2}, S_{1}, S_{5}, S_{10} S_{11} S_{23}$, and $S_{13}$ were detected. The other bands obtained from the PCR were related to the known self-incompatibility alleles that might be considered as new alleles. In the study population in this research, $S_{1}, S_{2}, S_{3}$, and $S_{11}$ alleles had higher frequency.
\end{abstract}

Keywords: molecular marker, allele, self-compatibility, PCR, almond

\section{Introduction}

In modern gardening, the percentage of adequate fruit development is highly important. Only at the time of optimal conditions for pollination and fruit development in the trees can a high yield be expected. Detecting self-incompatibility alleles is important for the proper selection of pollinizer trees and improving the yield in commercial gardens and also in improvement works to ensure success in the controlled crosses (Valizadeh et al., 2007). The almond (Prunus dulcis) belongs to the Rosaceae family and Prunoideae subfamily. The wild almond cultivars have been originated from natural masses in the Central Asia and are native to the dry mountainous regions of Central and Western Asia, extending to the Mediterranean coast. Today, the major areas of almond culture are concentrated in three parts of the world, including Asia, Mediterranean region, and the United States (State of California), but it is also cultivated at limited levels in Australia, 
South Africa, Argentina, and Chile (Gradziel \& Kester, 1996). Almond is one of the most important trees in temperate regions and is cultivated in different countries due to their nutritional value as an important and economic product. The quantitative and qualitative increase in almond products is one of the objectives of the research programs in most producing countries. An almond production problem is self-incompatibility, which is one of the important points in the almond breeding (Ebadi et al., 2011). The self-incompatibility in almonds is considered as a gametophytic genetic phenomenon. In this type of incompatibility, the growth of pollen tube is stopped in the middle style. The stop is due to the glycoprotein-like ribonucleases known as (S-RNases). This trait is controlled in almonds by a multi-allelic genetic locus (S-locus). The presence of similar alleles in pollen grains and pistil prevents the pollen tube from reaching the ovary and causes a cessation in the middle of the style. Today, about 32 types of $S$ alleles, known as $S_{1}, S_{2}, S_{3}, \ldots \ldots .$. $\mathrm{S}_{\mathrm{n}}$ are detected in almond and the $\mathrm{S}_{\mathrm{f}}$ allele has been introduced as the source of self-compatibility in almonds (Ortega and Daiesenta, 2008). Self-incompatibility prevents self-fertility, which is considered an advantage in the evolution, because it increases the interspecific crosses (Ortega and Daiesenta, 2008). However, this physiological phenomenon reduces the almond yield in the single cultivar. Therefore, in order to construct commercial almond gardens, at least two compatible cultivars are essential. Therefore, the detection of self-incompatible alleles (S-alleles), especially in the superior genotypes, is important for determining the pollinator cultivars. Nowadays, various methods have been invented for detecting genotypes and self-compatible cultivars from self-incompatible cultivars as well as determining $\mathrm{S}$ alleles in almonds, including the following methods (Kamali et al., 2009).

1. Branch coverage(Bagging)

2. Studying growth of pollen tube using fluorescence microscope

3. Analysis of Ribonucleases of flower style by NEPHGE

4. PCR method

PCR is used as a precise and new molecular method for detecting self-incompatibility in almonds and, today, with respect to its high precision and ease of use, it is used more than the analysis of style ribonucleases. The basis of this method is to use the specific multi- or single-allelic primers, which will proliferate a part of DNA chain, i.e. the corresponding allele (Chonon Tapitat et al., 2001: Chonon Tapitat et al., 2003: Ma and Olivier, 2001: Tamura et al., 2000). Other researchers have used a variety of specific primers to determine $S_{1}, S_{8}, S_{5}, S_{9}, S_{10}, S_{23}, S_{2}, S_{7}$, and $S_{f}$ alleles and succeeded in confirming some alleles or introducing new alleles (Chanon Tapitat et al., 2003). Today, many primers are known for detecting almond alleles, including 16 pairs and a single primer, making up 33 (Alonso and Socias Company, 2005). So far, many studies have been conducted on the self-incompatibility of almond in Iran (Ebadi et al., 2011: Sheikh Alyan et al., 2010: Zain al-Abedini et al., 2012, Kamali et al., 2009: Valizadeh Kaji et al., 2007). However, over these years, no self-incompatibility study has been conducted on the almonds in Central Iran, Yazd, and this led us to examine the almond genotypes in these areas. Using the markers applied in this research, it is possible to detect the self-incompatibility alleles in the almond genotypes and the self-compatible alleles can also be found among the studied almond genotypes.

\section{Materials and methods}


The leaf samples used in this research included 100 almond genotypes with good crops in recent years that have been collected from Iran's central plateau. It should be noted that most of the prepared samples were hard- and soft-shell, late-flowering, frost-resistant, and prolific genotypes. For the experiments, 5-7 leaves were collected from different young branches of different almonds genotypes and quickly transferred to the laboratory under cold conditions at the temperature of minus $20^{\circ} \mathrm{C}$. The second samples that were considered as the control consisted of 7 self-compatible hybrid almonds that were studied in terms of the allelic composition. In order to start the application of markers, the DNA extraction from the young leaf samples in these genotypes was carried out using the method by Gept and Clegg (1989). In this method, the sample was placed in a micro tube and $200 \mu \mathrm{l}$ of the extraction buffer of CTAB, including $100 \mathrm{mM}$ Tris hydrochloride, $2 \mathrm{mM}$ EDTA, $1.4 \mathrm{mM}$ NACL, $2 \%$ CTAB, $1 \%$ PVP, and $0.1 \%$ Iodide sulfide was poured into the micro tubes to completely cover the leaf samples. Afterwards, the micro tubes were frozen in the freezer and their content was crushed in a frozen state by a sterile instrument; the rest of the extraction steps were carried out inside the micro tube and DNA of the leaf sample was extracted. In order to extract the DNA in this experiment, mortar was not used. After completion of the extraction, the purity of DNA was evaluated by the Electrophoresis with 1.5\% Agarose gel. The Nano drop machine was used for the initial examination of quantity and contamination to phenolic and protein. The spectrophotometer was used at the wavelength of 260 to 280 for determination of purity and contamination of DNA. Finally, the concentration of DNA was reported in $n g / \mu 1$.

From among the experimented primers, several primers including 1- AS1II-AmyC5R 2- ConFConR 3-Cebador2-Cebador8 primers pairs were used for PCR. The sequence of each primer is shown in Table 1. All the primers have been used by other researchers to detect almond alleles (Ma and Olivier, 2001; Tamura et al., 2000). The sequence of primers is as follows:

Table 1- Primers and alleles detected in PCR

\begin{tabular}{|c|c|c|}
\hline Primer name & Primer sequences & Detectable alleles \\
\hline AS1II & TATTTTCAATTTGTGCAATGG & $\mathrm{S}_{1}, \mathrm{~S}_{\mathrm{f}}, \mathrm{S}_{3,}, \mathrm{~S}_{11}, \mathrm{~S}_{13}$ \\
\hline AmyC5R & CAAAATACCACTTCATGTAACAAC & \\
\hline ConF & GTGCAACAATGGCCACCGAC & $\mathrm{S}_{1}, \mathrm{~S}_{2}, \mathrm{~S}_{23}, \mathrm{~S}_{31}, \mathrm{~S}_{\mathrm{f}}, \mathrm{S}_{3}, \mathrm{~S}_{11}$ \\
\hline ConR & TACCACTTCATGTAACAACTG & \\
\hline Cebador2 & TACCACTTCATGTAACAACTG & $\mathrm{S}_{\mathrm{f}}$ \\
\hline Cebador8 & ACCACCTGCAGATTTAGCGGC & \\
\hline
\end{tabular}

The polymerase chain reaction was conducted by Thermocycler manufactured by PEQLAB, Germany. The temperature was considered from 55 to $62^{\circ} \mathrm{C}$. Depending on the anneling temperature of each primer, the PCR program was regulated. For PCR reaction, the master mix 
(5x (FIREPOL whit 7/5 Mm Mgcl2) was used in accordance to the instructions and in the final volume of $20 \mu \mathrm{L}$. The PCR mixture contained FIREPOL Master Mix (4 $\mu \mathrm{l})$, primer $(2 \mu \mathrm{l}$ each), and DNA $(1 \mu \mathrm{L})$. The final volume of the mixture per micro tube reached $20 \mu \mathrm{L}$. The PCR cycles consisted of an initial denaturation cycle for $3 \mathrm{~min}$ at $95^{\circ} \mathrm{C}, 35$ cycles containg, denaturation for $50 \mathrm{sec}$ at $95^{\circ} \mathrm{C}$, annealing of primers for $1 \mathrm{~min}$ at 55 to $60^{\circ} \mathrm{C}$, extension at $72^{\circ} \mathrm{C}$ for $50 \mathrm{sec}$, followed by final extension at $72^{\circ} \mathrm{C}$ for $8 \mathrm{~min}$. It should be noted that PCR mixture used in this research contained the loading buffer and PCR products did not need the addition of a heavy buffer. The PCR products were separated by the electrophoresis of $1 \%$ agarose gel in a TAE buffer $(0.5 \mathrm{X})$ at the voltage of $90 \mathrm{~V}$ for $1 \mathrm{~h}$. The gel staining was carried out at the stage of gel preparation using Green Viewer and there was no need for Ethidium bromide. Then, under the ultraviolet light (UV), the gel imaging was carried out using the gel documentation system. The size of the bands was recalculated by generic and specific primers in the studied genotypes using a 10-kg ladder .

\section{Discussions and Results}

According to the results of the PCR genotypes, these genotypes were divided into two selfcompatible and -incompatible groups. It should be noted that 32 studied samples were hybrid almond genotypes that had been obtained from the crossing of self-incompatible and -compatible cultivars. Out of 32 hybrid genotypes, 6 self-compatible genotypes were detected and considered as the control for self-compatible $\mathrm{S}_{\mathrm{f}}$ allele. The remaining self-incompatible genotypes were detected, in which the paired self-incompatibility alleles were detected. By calculating the size of the bands in the genotypes of the studied almonds and comparing them with the size of the bands associated with the pre-identified self-incompatible alleles, the genotype of self-incompatibility genotypes was determined. To this end, the AS1II and AmyC5R primer pair was used. After using this primer, it was expected to obtain the multiplied bands with the size of 1200 base pairs and, using this primer, 6 self-compatible samples were detected from 32 hybrid samples. These samples were considered as the self-compatible control sample (Figure 1).

Figure 1: Using AS1II- AmyC5R primer pairs for the detection of $\mathrm{S}_{\mathrm{f}}$ allele

Due to the proximity of the size of $S_{f}$ allele (1205 bp) $\left(S_{f}\right)$ and $S_{3}$ allele (1196 bp) $\left(S_{3}\right)$ with only 9 bp of difference, the bands were very close and sometimes mistakes might have occurred in the detection (Kamali, 2008). Hence, at the next stage of the experiment, a specific primer was used to detect the $S_{\mathrm{f}}$ allele so as to distinguish $\mathrm{S}_{\mathrm{f}}$ allele from $\mathrm{S}_{3}$ allele. For this purpose, the Cebador2 and Cebador8 specific primers were used to detect $S_{\mathrm{f}}$ allele. After using this primer pair, the bands of $1200 \mathrm{bp}$ were obtained from the bands created on Agarose gel that were related to the $\mathrm{s}_{\mathrm{f}}$ allele. Thus, using this primer pair, the self-compatible genotypes were distinguished from the selfincompatible ones (Figure 2). 
Figure 2: Cebador2-Cebador8 specific primer in the detection of $\mathrm{S}_{\mathrm{f}}$ allele in the self-compatible hybrid samples

In order to detect self-compatible and -incompatible alleles in almond genotypes, the AS1II (Forward) and AmyC5R (Revers) primer pair was used. In this study, using the aforementioned primer, the $S_{2}, S_{3}, S_{11}, S_{10} / S_{5}, S_{13}$, and $S_{23}$ alleles were detected. In addition to the above bands, a band of $750 \mathrm{bp}$ was seen that might be related to the new incompatibility alleles (Figure 3).

Figure 3: PCR results on Agarose gel: Using AS1II-AmyC5R primer pairs

Detecting $\mathrm{S}_{1}, \mathrm{~S}_{2}, \mathrm{~S}_{3}, \mathrm{~S}_{11}, \mathrm{~S}_{13}, \mathrm{~S}_{5} / 10$, and $\mathrm{S}_{23}$ alleles

The primer pair consisted of the ConF primer (Forward) and the ConR primer (Reverse ). The size of the bands resulting from PCR products on the Agarose gel was follows: 1100, 800, 1200, 600, 700,978 , and 792 base pairs that were respectively related to $S_{1}, S_{2}, S_{3}, S_{10}, S_{11}, S_{23}$, and $S_{31}$ alleles. Furthermore, bands with the size of 900 and 1000 primer pairs were also found, which did not belong to any of the known $\mathrm{S}$ alleles and might be related to new self-incompatibility allele (Figure 4).

Figure 4: General amplification of self-incompatibility alleles in the studied genotypes by PCR method using the ConF-ConR marker

In this experiment, by examining the results of PCR on the Agarose gel, 7 types of selfincompatibility alleles were detected. By investigating the size of the bands obtained from PCR of each DNA, the allele pair for each genotype was determined. In this study, a total of 9 selfincompatibility alleles, including $\mathrm{S}_{1}, \mathrm{~S}_{2}, \mathrm{~S}_{3}, \mathrm{~S}_{5}, \mathrm{~S}_{10}, \mathrm{~S}_{11}, \mathrm{~S}_{13}, \mathrm{~S}_{23}$, and $\mathrm{S}_{31}$ alleles were identified in the examined genotypes. By studying the number and type of the identified alleles in each genotype, the frequency of each allele in the population was determined. In the meantime, the $S_{1}$, $\mathrm{S}_{2}, \mathrm{~S}_{3}$, and $\mathrm{S}_{11}$ alleles had higher frequency. The frequency diagram of alleles is shown in Figure 5 .

Figure 5: Frequency diagram of the $\mathrm{S}$ multiplied alleles in the studied population Discussion 
The mortar was not used to grind the leaves for DNA extraction in these experiments and the ease of work was impressive. Furthermore, no contamination was seen in the tested samples and these results were compatible with the results by Kamali et al. and Hamidi et al. (Kamali et al. 2011; Hamidi et al. 2013). Given that at the beginning of the experiment, the DNA extraction of the almond samples was done using the method of Gept and Celegg (1989), during the DNA extraction process in this method, the liquid nitrogen was not used. It can be said that this was an appropriate method for extraction and the efficiency of this method was confirmed in this study (Ebadi et al., 2011). The efficiency of the ConF-ConR primer pair was confirmed for the detection of $S_{1}, S_{3}$, $\mathrm{S}_{11}$, and $\mathrm{S}_{\mathrm{f}}$ alleles (Channuntapitat et al., 2001; Ebadi et al., 2011). Using the above-mentioned primer pair, the $S_{31}, S_{10}, S_{2}$, and $S_{23}$ alleles were also detected. The detection of these alleles by primer pairs has not been reported in other references and was reported for the first time in this study. The $S_{31}$ allele is a self-incompatible allele identified as a new allele, which has not been reported in scientific references in Iran. In this study, the above allele was observed and reported among the almond genotypes in Yazd province of Iran. Further studies are required for proof. In order to detect this allele, specific primers should be designed and constructed, so that this allele can be determined definitively.

Another primer pair, i.e. AS1II-AmyC5R, was confirmed for detecting $S_{1}, S_{2}, S_{3}, S_{5} / 10, S_{11}, S_{13}$, and $S_{23}$ alleles (Ebadi et al., 2011; Sheikh-Alian et al., 2010; Zeinalabedini et al., 2010; Tamura et al., 2000; ValizadehKaji et al., 2007; Zeinalabedini et al., 2012). The results showed that, although the use of this primer pair (AS1II-AmyC5R) led to similar results in the generation of desired bands, the resulting bands were not as clear as those of previous primers. According to the results of these researchers, the bands made for $S_{3}$ and $S_{f}$ alleles were of the same size; thus, it is better not to use this primer pair to detect $S_{\mathrm{f}}$ and $S_{3}$ alleles. In this experiment, this result was also approved. In addition to the use of primer pair Cebador2-Cebador8 in this research for the initial detection of self-compatible and -incompatible genotypes, similar results for the SfF-SfR primer pair were consistent with the results by Kamali et al. in terms of detecting the self-compatible and -incompatible genotypes (Kamali et al., 2009). Finally, according to the results of this study, other studies can hybridization of the detected self-compatible genotypes with native self-incompatible genotypes of Yazd Province and provide self-incompatible genotypes in the region, thus increasing the almond yield in Yazd Province by cultivating self-compatible genotypes in order to overcome

\section{References:}

1. Amir Ghasemi T (2002). Almond processing, plant, production.Posterity, Tehran.

2. Alonso JM Socias i Company R (2005).Identification of the self-incompatibility allele in almond by specific primers.Journal of Agricultural Research. 3: 296-303.

3. Channuntapipat C, Sedgley M, Collins G (2001). Sequences of cDNAs and genomic DNAs encoding theS1, S7, S8 and Sf alleles from almond, Prunusdulcis. Theor. Appl. Genet. 103:11151122.

4. Channuntapipat C, Wirthensohn M, Ramessh S, Batlle A, Arus I, edgley P, Collins G (2003). Identification of incompatibility genotypes almond (Prunusdulcis Mill.) using specific primers based on the introns of the S-alleles. Plant Breeding, 122: 164-168. 
5. Ebadi A, kamali K, FatahiMoghadam R,. Naghavi M.R, Imani A (2011). Determination of Self-Compatible Almond Genotypes Obtained from Controlled Crosses and Identification of SAlleles in some Foreign Cultivars and Genotypes by PCR. Iranian Journal Seed and Plant Improvement, 27-1(1): 57-67.

6. Gept P, Clegg M (1989). Genetic diversity in pearl millet (Pennisetum glaucum) at the DNA sequence level.Journal of Heredity, 80: 203-208.

7. Gradziel T.M, Kester D.E (1996). Genetic improvement. pp. 70-75. In: Micke, W. C. (ed.). Almond Production Manual.Division of Agricultural and Natural Resources, University of California, Oakland, CA, USA.

8. Imani A (2000). Almond Breeding, Agricultural Extension and Education, Tehran.

9. Sheikh-Alian A, Vezvaei A, Ebadi A, Fatahi-Moghadam M.R, Sarkhos A (2010). Determination and identification of self-incompatibility alleles in selective Iranian and foreign almond cultivars (Prunus dulcis M.) by PCR Method. Iranian Journal of Horticultural Sciences, $41: 247-252$.

10. Zeinolabedini M, Khayam-Nekoui M, Imani A, Majidian P (2012). Identification of selfcompatibility and self-incompatibility genotypes in almond and some Prunus species using molecular marker. Iranian Journal Seed and Plant Improvment, 28-1(2): 227-238.

11. Kamali K (2008). An investigation of classical and molecular methods in almond breeding program to produce self-compatible genotypes. Ph.D. Thesis. Tehran University. Tehran, Iran.

12. Kamali K, Ebadi A, FatahiMoghadam R, Naghavi M.R, Imani A (2009). Heritability of $\mathrm{S}_{\mathrm{f}}$ Allele in Almond Progenies with PCR Method. Iranian Journal of Horticulture Sciences, 40: 6168 .

13. Ma R.C, Olivier M.M (2001). Molecular cloning of the self-incompatibility genes S1 and S3 from almond (Prunusdulcis cv. Ferragnes). Sex Plant Reprod. 14:163-167.

14. Ortega E, Dicenta F (2003). Inheritance of self-compatibility in almond: Breeding strategies to assure self-compatibility in the progeny. Theoretical and Applied Genetics, 106, p. 904-911.

15. Ortega E, Dicenta F (2008).Inheritance of self-compatibility in almond.Theoretical and Applied Genetics106: 904-911.

16. Tamura M, Ushijima Sassa Hirano H, Tao R, Gradziel T.M, Dandekar A.M (2000).Identification of self-incompatibility genotypes of almond by allele-specific PCR analysis. Theoretical and Applied Genetics.101:344-349.

17. ValizadehKaji B, Ershadi A, Gholami M (2007). Identification of self-incompatibility alleles in some Iranian and forign almond (PrunusdulcisM.) cultivars using PCR.Iranian Journal of Horticultural science and Technology, 8(4), 249-258. 
18. Zeinalabedini M, Khayamnekue M, Imani F, Majidian P (2012). Identification of SelfCompatibility and Self-Incompatibility Genotypes in Almond and some Prunus Species Using molecular marker. Iranian Journal Seed and Plant Improvement, 27-1(1): 57-67. 